\title{
RELIABILITY INSPECTION OF ELECTRONIC COMPONENTS FROM THE POINT OF VIEW OF THE USERS
}

\author{
F. BRÁDA, CS. FOZO, I. GÖBLOS \\ Industrial Research Institute for Electronics, Budapest, Hungary, "HIKI".
}

(Received April 16 1981; in final form October 20, 1981)

\section{INTRODUCTION}

One group of electronic components, passive components, has a mature production technology, since they have been in production for a long time. On the other hand in another group of electronic components, such as microcircuits, the manufacture is being continuously changed as new physical phenomena are recognised. The production technology of this latter group can only be developed on the basis of a carefully planned chain of production and inspection phases based on very sensitive and precisely adjustable equipment.

Amongst the main objectives of the componentmanufacturers who have a mature production technology the reliability assurance of their products has a very important role. Consequently, the manufacturing process is based on a well-organised chain of operation, inspection and surveillance. The number and severity of the inspections and surveillances built in between the different manufacturing operations can vary depending on the component-type.

Great attention should be paid to the reliability of electronic components, since reliable equipment cannot be constructed without reliable parts. Consequently, the satisfactory reliability level of components is important in the economic consideration of the component-manufacturers and of the users, i.e. the equipment-manufacturers, as well. It is important for the component-manufacturer, to ensure that their products will have a good market, and it is important for the user because he must produce reliable equipment.

In the following paper the activities performed to achieve these two objectives will be discussed. In the first part a brief summary will be given of the evaluation process, whilst in the second part illustrative examples of testing procedures will be given.

\section{ASSURANCE OF THE RELIABILITY LEVEL OF ELECTRONIC COMPONENTS}

The reliability assurance of electronic components is unambiguously considered as a task of the componentmanufacturer. The field can be discussed on the basis of the following two aspects.

\subsection{Production of a Consistent Quality}

For economical and technical reasons, the component manufacturers are aware of the fact that their production technology should be maintained at an acceptable level. In particular, economical considerations force manufacturers to investigate new suitable technical solutions to production problems and to put these technical solutions into practice.

Manufacturers are at all times making efforts to:develop new products;

develop and establish production technology at a level that is satisfactory both from the technical and quantitative points of view;

apply thoroughly specified raw materials of a consistent quality;

meet the storage and transport requirements.

These aspects should be emphasised, although the detailed discussions of these problems are not presented here, because it is well known that reliable components cannot be produced if these basic principles are neglected.

\subsection{Reliability Tests Performed by the Component- Manufacturers.}

Component manufacturers are always making efforts to demonstrate the reliability of their products through the presentation of the reliability test results and with the reliability characteristics derived from the test results. In order to obtain reliability data, the manufacturers will have performed tests during the production process and also reliability tests under laboratory conditions.

In the tests performed by the manufacturer during the production process, economical considerations are all important. Therefore with a view to economy, it is required that the tests must be carried 
out in as short a time as possible. Also test results must be available on each phase of production in order to be taken to prevent any additional cost due to the completion of non-conforming of semi-finished parts.

\section{DATA AVAILABLE FOR USERS}

If no other agreement was reached between the user and the producer with regard to the quality of the components, there will only be available data published in the manufacturers catalogue. Nowadays this data gives detailed information, but is often not sufficient to meet the requirements of the users.

At the present time the manufacturers are producing components of different quality grades. The componentparameters/electrical parameters have been measured and on the basis of this a quality classification has been made. In addition, data on LTPD or AQL-values and failure criteria is made available. In order to assess the reliability of components, however such data needs to be supplemented by information on the parameterdrifts of the components occurring as a result of applied stresses. Tests of this kind that are performed include: thermal cycling, centrifuge, bumping, vibration, humidity tests, storage at high and low temperatures and stabilization baking.

\section{TESTS PERFORMED BY THE USERS}

The equipment manufacturers, i.e. the users of the electronic components, are mainly guided by economical considerations, when they determine the components to be used in their equipment. Components are selected which satisfy their needs from the two points of view of price and quality.

There is no doubt that the equipment manufacturers would be best served if only totally reliable components were available.

Since, in the production of a complex equipment with a large number of components it is more difficult to prevent failures caused by components operating unsatisfactorily, the quality of the components must be controlled in some way. (For instance, on the basis of field data it was observed that telecommunication equipment with 900 components had a Mean Time Between Failure (MTBF) of 50,000 hours, while other telecommunication equipment with 2250 components had MTBF equal to 20,000 hours.) It follows from the above mentioned reasons that components assessment must not be restricted only to the measurement of electrical parameters.
The application of screening tests that are well-known in the international literature should be performed $(1,2)$. Since these tests are expensive and time is required to perform them, a reasonable grouping of the tests is necessary. In the arrangement of the tests it is necessary to start from the fact that the tests should accelerate the failure mechanism characterising the component-type $(3,4,5)$ and should provide information as to the early failures in components. As examples of user tests, LEDS and memory systems will now be considered.

\subsection{Testing of LEDs}

As a simple method of test, visible radiation detection from LEDs can be mentioned. As a consequence of positional deviations resulting from the construction of these diodes, they may have defects which result in an innacurate evaluation, if the LEDs are applied in the field. Consequently, it is reasonable to test the deviation of the luminous intensity on the basis of a geometrical arrangement, i.e. on the basis of the deviation of the optical and geometrical axis. This can be tested by means of an optical bench, but

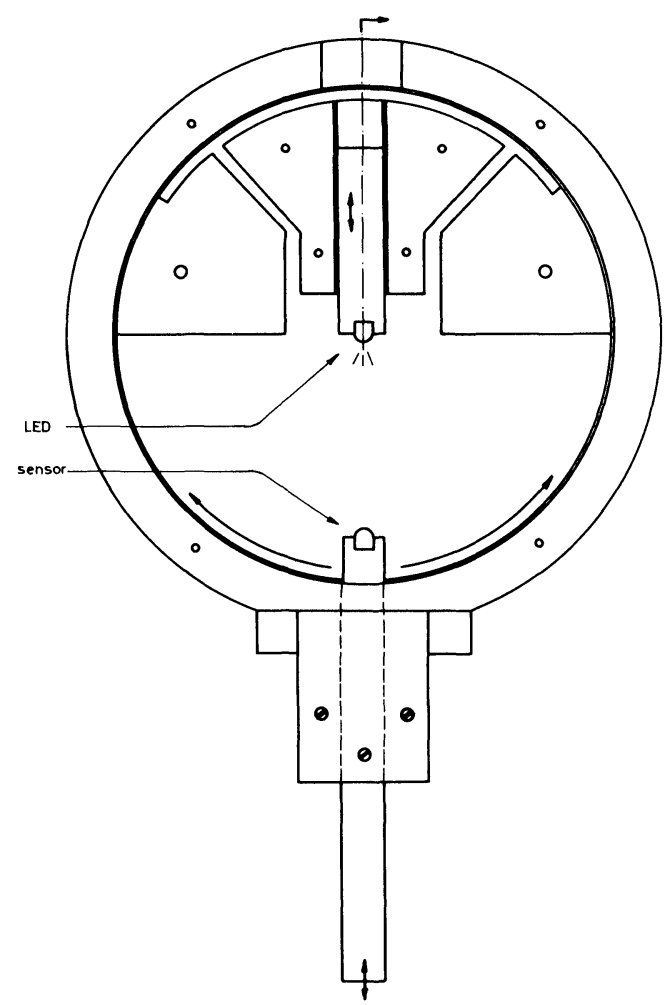

FIGURE 1 Mechanical mounting arrangement for the determination of the radiation characteristics. 
in this case a special amplifier is required for detection. An optical bench is not necessarily required for the aligning of the diode, since this can be accomplished by a simple mechanical mounting, if the required precision and reproducibility can be assured. Since the LEDs radiate into space, an arrangement is constructed which allows of the determination of the radiation characteristics in two planes perpendicular to each other. (Figure 1.) A photodiode having a high sensitivity, (such as BPW 24), should be used for the measurement. As a characteristic to be measured, the short circuit current of the photodiode, or a voltage proportional to it, should be chosen which is a linear function of the illumination. In order to allow of the measurement of the voltage in the sensitivity range of a DVM, the voltage of the photodiode must be amplified. Since a low voltage level can be observed in the input of the measuring amplifier, the offset voltage and the error signal coming from the photodiode should be compensated for with an automatic zero adjust circuit. Compensation is obtained with a feedback loop containing a sample-holder. The sensing amplifier can be developed as a complete measuring unit containing a current-source to drive the lightsource. The current-source is designed so that it will be able to generate the pulse shapes frequently used in practice.

As a practical application of the method presented above, it should be emphasised that the measuring arrangement discussed is suitable not only for testing LEDs, but for the testing of sensing diodes, provided a reference light-source is placed in the position of the LED. The measuring system is suitable for determining the following characteristics:

- radiation characteristics of LEDs,

- the luminous intensity measured in the direction of the case axis,

- the electrical characteristics.

In order to take into consideration the aspects of use, tests can be performed to determine the following data:
- the comparison of the radiation intensities in the direction of the case axis for devices produced by different manufacturers,

- the differences in the electrical parameters,

- the changes in the optical and electrical characteristics occurring on the effect of stresses.

From measurement it can be observed that the parameters of the LEDs produced by different manufacturers are very different, although they are recommended for the same application and they have the same sizes (see Table I).

To illustrate the statements mentioned above, test data are summarised in Table 1 for LEDs produced by four different manufacturers (denoted by letters A, B, C, D, respectively). In the first column of Table 1 the ratio of the mean radiation intensities between the devices of manufacturers $B, C, D$, respectively, and the manufacturer $A$ can be seen. In the second column the angles of the deviations between the optical and case axis are presented. Finally, in the third column the mean values of relative radiation intensity changes in percentages which were observed after 1000 hours at stress conditions $I_{F}=50 \mathrm{~mA}$; $\mathrm{T}_{\mathrm{A}}=25^{\circ} \mathrm{C}$ are given for the devices of manufacturers A, B, C, D.

The values of the angles of the maximum radiation can vary in the range $30-120^{\circ}$.

A considerable difference can be observed in the radiation intensity measured in the direction of the case axis for different specimens of the same device type. In the radiation intensity a spread of 1.3-2.6 times can be found in the case of devices produced by different manufacturers. It follows that LEDs from different producers cannot be used in the same field, since it would result in an inaccurate evaluation. Deviation of the magnitude is admissible on the basis of catalogue data, but specimens having deviating characteristics may cause faulty perception, if they are used in the same environment, such as in a LEDfield.

TABLE I

Test Results of LEDs for Four Different Manufacturers;

Test Conditions: 1000 hours; $\mathrm{I}_{\mathrm{F}}=50 \mathrm{~mA} ; \mathrm{T}_{\mathrm{A}}=25^{\circ} \mathrm{C}$

\begin{tabular}{llll}
\hline $\begin{array}{l}\text { Symbol of the } \\
\text { manufacturer }\end{array}$ & $\begin{array}{l}\text { Ratio of radiation intensities } \\
\left(\mathrm{E}_{\mathrm{k}} / \mathrm{E}_{\mathrm{A}}, \mathrm{k}=\mathrm{A}, \mathrm{B}, \mathrm{C}, \mathrm{D}\right)\end{array}$ & $\begin{array}{l}\text { Deviation angle between the } \\
\text { optical and case axis }\end{array}$ & $\begin{array}{l}\text { Mean relative radiation intensity } \\
\text { change } \Delta \mathrm{E}(1000) / \mathrm{E}(0)\end{array}$ \\
\hline $\mathrm{A}$ & 1.0 & 0 & $-20.4 \%$ \\
$\mathrm{~B}$ & 2.60 & $\pm 5^{\circ}$ & $-16.3 \%$ \\
$\mathrm{C}$ & 2.43 & $\pm 15^{\circ}$ & $-19.0 \%$ \\
$\mathrm{D}$ & 1.30 & 0 & $-14.4 \%$ \\
\hline
\end{tabular}




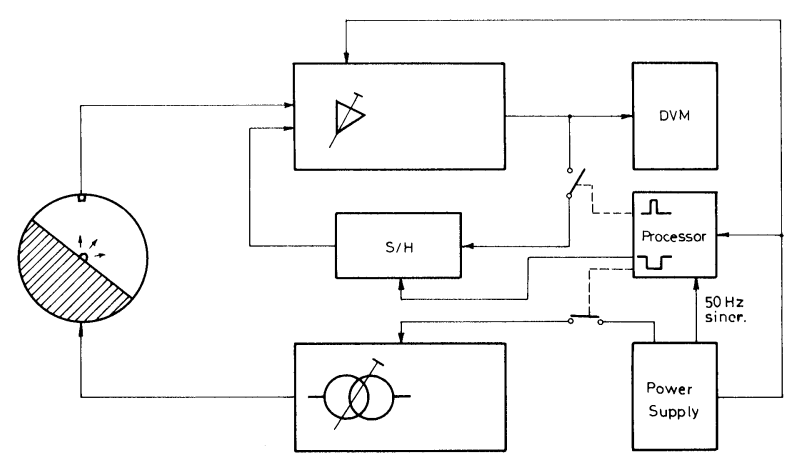

FIGURE 2 Blockdiagram of a measuring arrangement for testing LEDS

It should be noted that the acceptance of LEDs is based on the catalogue data of the manufacturer, but the use of these devices requires more severe requirements for the parameter drifts. Therefore, it is necessary to test the LEDs as to whether they meet the requirements for parameter drifts lower than the values prescribed in catalogue.

The above mentioned method can be used for the purpose of measurement. In addition, for the inspection of the basic parameters, it is necessary to perform screening tests such as are applied to components, such as ICs.

The testing methods must be selected with a knowledge of the device construction and technology. On the basis of experience the most effective test sequence has been found to be as follows: rapid temperature change, stabilization bake 24 hours, functional test performed with the measuring equipment discussed.

In the screening tests the following dominating failures can be observed: bonding failures, chipfailures (radiation intensity is decreasing). It should be noted that the majority of chip failures occur in the first period of the stabilization bake.

\subsection{Testing of memories.}

From a point of view of reliability, a particular testing technique is required in the case of some types of devices, such as PROM-s and REPROM-s, having operational properties developed by the users. The reliability of these devices is not influenced by the production technology only, but by the programming technique used also.

As is known, the physical basis of information storage, and consequently the programming technique, is quite different for bipolar and MOS PROM-s. ${ }^{6,7}$

In the case of bipolar PROMs the programming is performed by fusing a link (e.g. NiCr link) in a memory cell. ${ }^{7,8}$ This operation will considerably influence the field reliability of the memory. Besides the knowledge of technological failures, it is very important to determine which kind of failures can be observed due to the programming procedure.

Failure analysis should be performed to state the cause of the failure. On the basis of our test results, it can be stated that a departure from the energy range of the programming specified by the manufacturer can cause the failure of the program memory. ${ }^{7}$

For instance, in the case of PROMs type N82S23B, when the energy level exceeded the manufacturer's recommended value by $50 \%$, the fuse was melted and damage was observed in its neighbourhood (see Figure 3).

If an energy level lower than the recommended values (and their limits) is applied, this will result in omitting the information input. On the other side, if the energy level is higher than the recommended values this will result in damage to the chip in the 'fusing link'.

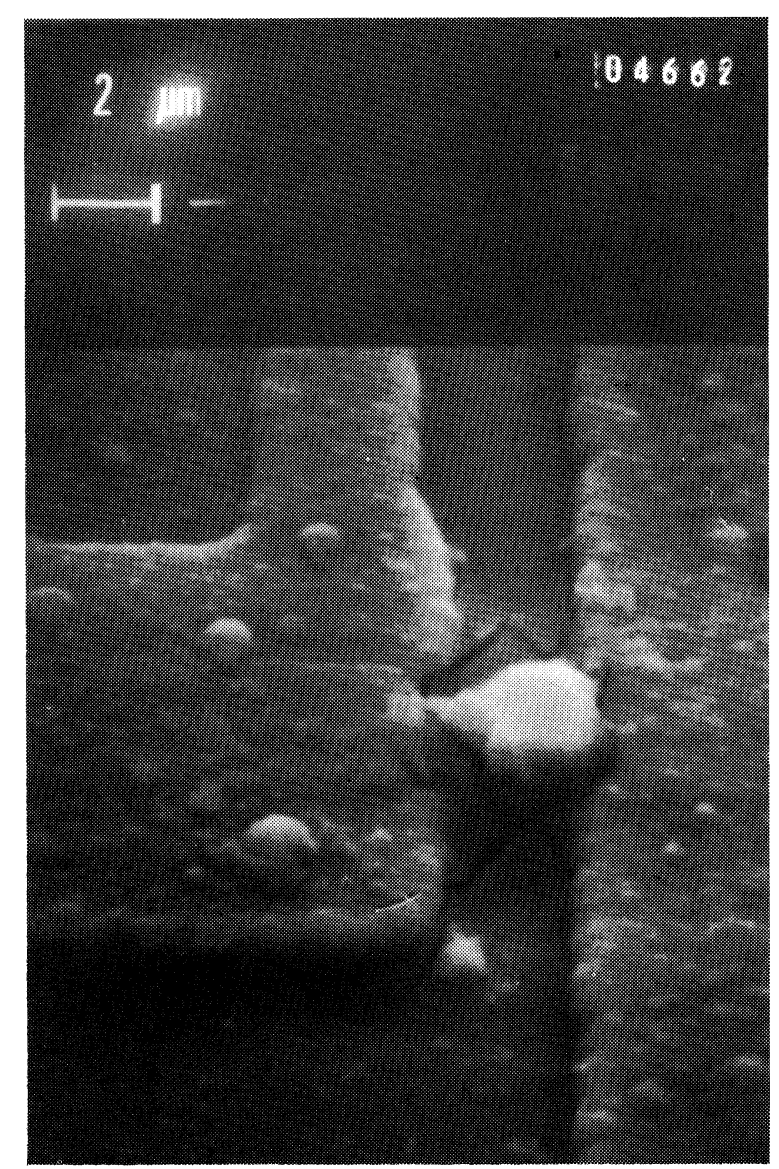

FIGURE 3 Damage in PROM cell due to a high energy level. 
TABLE II

$1000 \mathrm{Hr} ., 125^{\circ} \mathrm{C}$ Dynamic Lifetests on Silicon Fuse PROMs (see Ref. (9))

\begin{tabular}{lccl}
\hline Device & No. UNITS & & FAILURES \\
\hline 1 K PROM & 1000 & 0 & \\
2 K PROM & 900 & 0 & \\
2 K PROM & 300 & 2 & $\begin{array}{l}1 \text { marginally } \\
\text { programmed } \\
\text { fuse } \\
\end{array}$ \\
& & & $\begin{array}{l}1 \text { nonfuse } \\
\text { related }\end{array}$ \\
& & & \\
\hline K PROM & 350 & 0 &
\end{tabular}

It follows from the above mentioned facts that the tests of PROM-s should be carried out after fusing. On the basis of our experiences, it is necessary to perform dynamic tests on the PROMs with reading cycles in order to reveal the memory cells that have been fused in non-conformity with the specifications.

Test results to $1000 \mathrm{Hr}$, $125^{\circ} \mathrm{C}$ Dynamic Lifetests presented in (9) are given in Table II.

In the case of REPROMs a different testing technique is required, since the information storage is performed on the basis of the charge storage principle. In applications REPROMs should stand the effects of several programming and erasure cycles.

In case of a circuit using FAMOS memory cells for example, the information is the charge amount accumulated by the programming in the floating gate..$^{10,11}$ Consequently, the decrease or the loss of the charge amount results in an information loss. Therefore, it is reasonable to test the charge amount accumulated by the programming in the floating gate or the threshold voltage shift which is proportional to it. In such a way the precise adjustment and the continuous testing of the programming conditions can be assured.

The stored charge amount can be tested by observation of the charge loss process in time and a conclusion can be drawn for the storage capability of the cell. If a device has a higher time constant during erasure, it is expected that its spontaneous charge loss will occur after a longer time period.

The performance of programming and the erasure under controlled conditions and the application of a storage test at high temperature $\left(250^{\circ} \mathrm{C}, 168\right.$ hours) can be considered as an effective screening test.

On the basis of our test results it can be stated on the effect of the high temperature $\left(250^{\circ}\right)$, after 168 hours that about $10 \%$ of the stored charge had been lost in case of reliable cells, but for failed cells a total charge loss had been observed. Therefore, the storage test at high temperature is a very effective tool to screen the devices having failed cells. In addition, this test can be considered as an acceleration test, because an acceleration factor $7.10^{4}$ between the reliability characteristics at the temperatures $250^{\circ}$ and $70^{\circ}$ has been found. This acceleration factor is equivalent with an activation energy $1 \mathrm{eV}$ (see Ref. 3).

The test of memories as LSI devices cannot be restricted to a functional test and to the measurement of static parameters. They need to be tested as to whether the devices will perform the required function under the conditions of the actual environment. According to our experience, besides the application of the systems for measuring the parameters, it is necessary to develop and to apply relatively simple

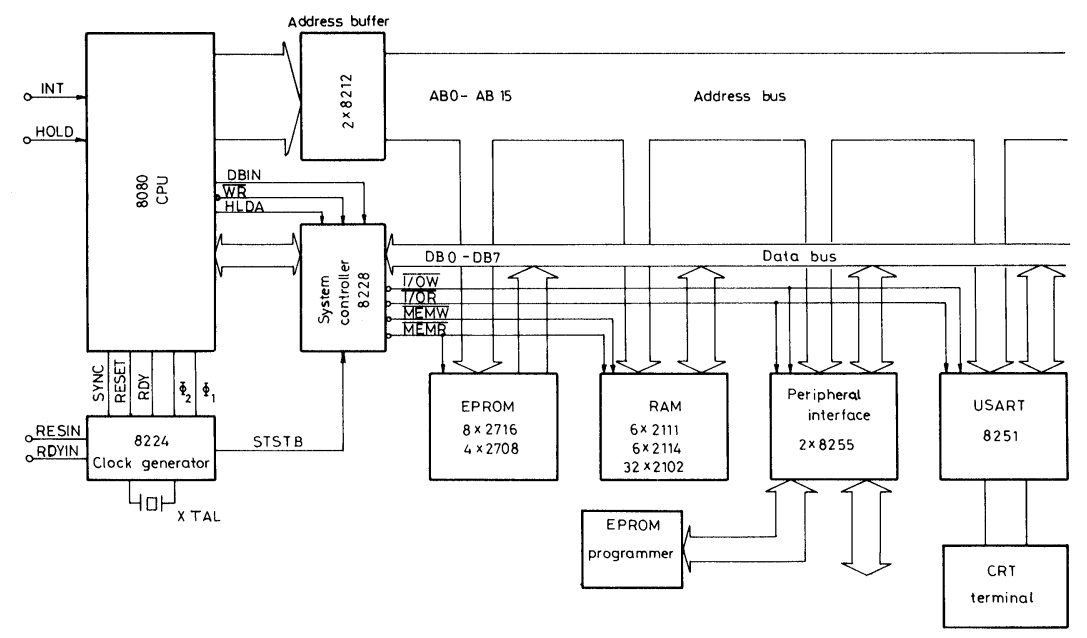

FIGURE 4 Blockdiagram of a simple testing arrangement. 
testing equipment which allows of the simulation of the actual operating conditions of the device. This equipment allows the testing of the dynamic parameters as well.

The solution of these tasks can be considerably simplified by the application of microprocessors. For example, a suitable system has been developed by the application of a $8080 \mathrm{CPU}$ having a memory capacity of 20 kbyte EPROM and of a 8 kbyte RAM. The circuit to be tested can be connected to the system by a peripherical interface (see Figure 4). The manmachine connection can be assured by a suitable display (Orion ADP 2000).

The system provides the following possibilities:

- the dynamic parameters of components important to the system operation can be obtained by the connection of external measuring units,

- the programming of EPROMs and testing of their programming conditions,

- running of programs developed for testing RAMs.

Comparing the system constructed in the above manner with the normally used measuring equipment, it can be stated that the above system is suitable for the determination of critical dynamic parameters and will provide information for the applicability of components produced by given manufacturers in the same system.

An additional advantage of the system is that the large memory capacity allows of the storage of a large number of test patterns for testing LSIs. Using properly selected peripherials and programs the system is suitable for the observation and registration of failures including software-failures.

\section{CONCLUSIONS}

As is known, testing systems specified in detail are available for electronic components. The manufacturer can produce their devices on the basis of a matured and precisely adjusted technology. The components are qualified from the point of view of reliability and quality.

On the basis of practical experiences, in addition to carefully organised component testing by the producer, it is necessary for the users to perform tests. The test conditions should meet approximately the same requirements as will be observed in the practical application of the devices. These tests are required even if the usually specified screening tests are performed and incoming-goods inspection is applied as well. Examples of such tests are given for LEDs and memory systems, and a detailed testing arrangement for LSI memory testing is discussed.

\section{REFERENCES}

1. Reliability Analysis Center (RADC), Reliability Design Handbook, RDH-376, (1976)

2. Reliability Analysis Center (RADC), Microcircuit Screening Effectiveness, RADC RAC Report TRS-1 (1978)

3. R.M. Alexander, Accelerated Testing in FAMOS $8 \mathrm{~K}$ EPROM, 15th Proc. IEEE, Reliab. Phys. Symp., pp 229-232 (1977)

4. B. Reich, A Study of Accelerated Storage Conditions Applicable to Semiconductor Devices and Microcircuits, IEEE Trans. Reliab. R-27, pp 178-180 (1978)

5. H.S. Blanks, The Temperature Dependence of Component Failure Rate, Microelectron. Reliab. Vol. 20, pp 297-307 (1980)

6. I.J. Chang, Nonvolatile Semiconductor Memory Devices, Proc. of the IEEE, Vol. 64, No. 7. (1976)

7. J.L. Davidson et al., Fusing Mechanism of NichromeThin Films, 14th Proc. IEEE, Reliab. Phys. Symp., pp 173-181 (1976)

8. G.B. Kenney et al., Mechanism of Nichrome-Linked Programmable Read-Only Memories, 14th Proc. IEEE, Reliab. Phys. Symp. 164-172 (1976)

9. R.C. Smith et al., Reliability Studies of Polysilicon Fusible Link PROMs, 14th Proc. IEEE, Reliab. Phys. Symp., pp 193-197 (1976)

10. D. Frohman-Bentchkowsky, A Fully Decoded 2048 Bit Electrically Programmable MOS Read-Only Memory, 1971 ISCC, Philadelphia, Feb. 17-19, 1971

11. G. Gear, FAMOS PROM Reliability Studies, 14th Proc. IEEE, Reliab. Phys. Symp., pp 198-201 (1976). 

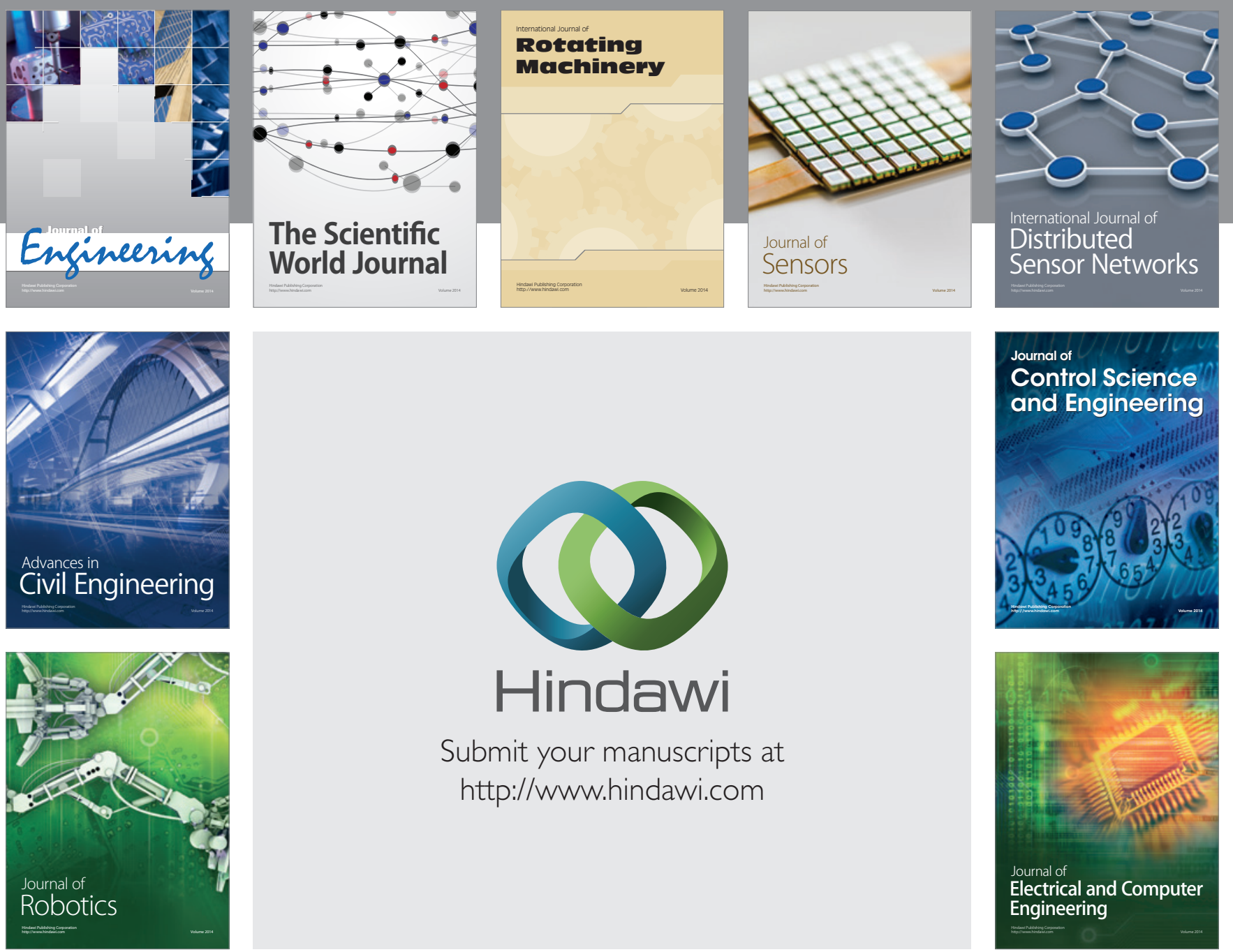

Submit your manuscripts at

http://www.hindawi.com
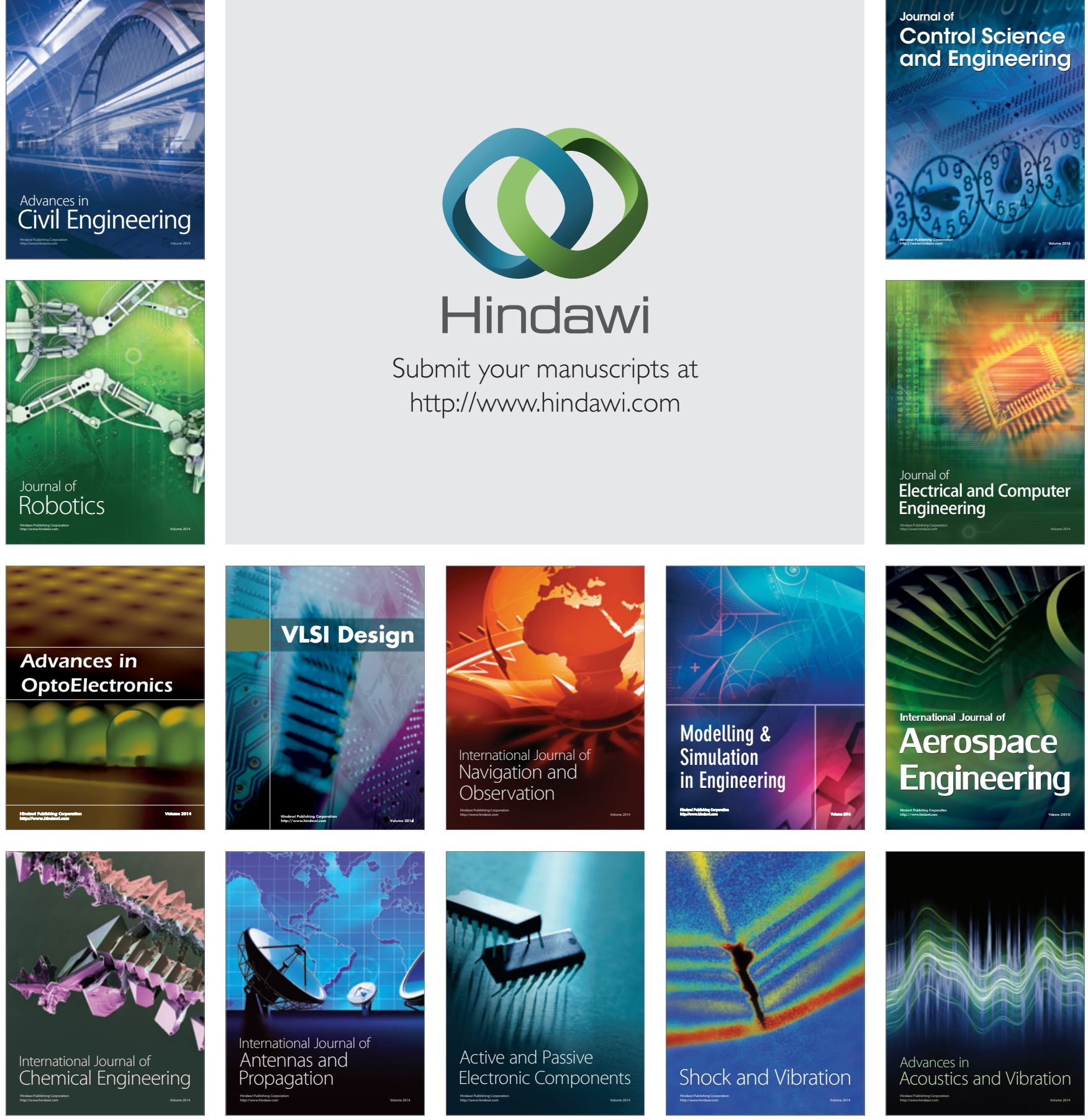